

OPEN

SUBJECT AREAS:

NANOSCIENCE AND

TECHNOLOGY

MECHANICAL ENGINEERING

APPLIED PHYSICS

CHEMICAL PHYSICS

Received

11 August 2014

Accepted

15 September 2014

Published

8 October 2014

Correspondence and requests for materials should be addressed to

Y.S. (syt06@mails. tsinghua.edu.cn)

\section{Time-dependent Gas-liquid Interaction in Molecular-sized Nanopores}

\author{
Yueting Sun ${ }^{1}$, Penghui Li ${ }^{1}$, Yu Qiao ${ }^{2}$ \& Yibing Li ${ }^{1}$ \\ 'State Key Laboratory of Automotive Safety and Energy, Tsinghua University, Beijing, 100084, P.R. China, ${ }^{2}$ Department of \\ Structural Engineering, University of California - San Diego, La Jolla, CA 92093-0085, USA.
}

Different from a bulk phase, a gas nanophase can have a significant effect on liquid motion. Herein we report a series of experimental results on molecular behaviors of water in a zeolite $\beta$ of molecular-sized nanopores. If sufficient time is provided, the confined water molecules can be "locked" inside a nanopore; otherwise, gas nanophase provides a driving force for water "outflow". This is due to the difficult molecular site exchanges and the relatively slow gas-liquid diffusion in the nanoenvironment. Depending on the loading rate, the zeolite $\beta$ /water system may exhibit either liquid-spring or energy-absorber characteristics.

F luidic behavior in nanoscale channels is of great interest due to its potential applications such as drug delivery $^{1}$, cellular probes ${ }^{2}$, nanofluidic chips ${ }^{3}$, and energy related applications ${ }^{4,5}$, etc. For instance, the pressure-induced intrusion of liquid into hydrophobic nanopores can contribute to a highly-efficient energy absorption system. By developing ultra-large solid-liquid interface, large amount of mechanical energy can be converted into interfacial energy ${ }^{4,6}$. Based on the plentiful molecular dynamic (MD) simulations and experiments conducted, it's found that nano-confined liquid behavior cannot be explained by conventional continuum theories, and exhibits unusual characteristics such as the exceptionally high transport rate compared with bulk fluid $^{7-12}$. However, current effort is limited to the liquid-solid interaction in nanopores, the effect of gas phase, i.e. the nanoscale gas-liquid interaction has not received adequate attention.

MD simulations are generally conducted on vacuum nanotubes ${ }^{8,9}$. For example, Hummer, et al. ${ }^{9}$ reported the spontaneous filling of a nonpolar carbon nanotube with water molecules and suggested carbon nanotubes to be molecular channels for water and protons. However, this finding may not apply under non-vacuum condition, which is normally the case in practical applications. Experimental works are generally conducted with the presence of gas phase, but they're mainly focused on the pressure-induced inflow process instead of the quasi spontaneous outflow behavior, so that the gas phase effect is diminished and left undiscussed. For example, Qiao et al. ${ }^{6,13,14}$ provided a number of experimental results on the pressure-driven liquid infiltration on nanoporous silica, carbon and zeolites, while the liquid outflow behavior as pressure is removed is not fully understood so far. For the limited experimental works involving nanoscale gas-liquid interaction, the length scale of nano-confinement is generally large, at least one order of magnitude higher than the size of water molecules, and thus they are still focused on the "continuum" topics, such as the gas-liquid interface (menisci shape) in nanotubes ${ }^{15,16}$, the nucleation and growth of gas phase $e^{17}$, and the gas solubility in nano-confined liquid ${ }^{18}$. Such results are unlikely to disclose the unique discrete nature expected in the molecular-sized nanopores (with pore size comparable with the size of water molecules). To date, no experimental observation has explored the gas-liquid interaction in molecular-sized nanopores.

In bulk liquid, as well as the liquid confined in relatively large nanotubes, gas has much lower density and higher compressibility compared with liquid, thus liquid-solid interaction plays the dominant role in fluidic behavior and gas phase effect is secondary. While under a molecular-sized nanoscale confinement, liquid and gas molecules will be arranged into a single-file chain ${ }^{9,19}$, and the effective density of gas molecules significantly increases. Gas molecules can thus make a noticeable contribution to the liquid motion. In this paper, we provide an experimental investigation on the gas-liquid interaction in molecular-sized nanopores, through an observation on the outflow of pressurized liquid in zeolites. It will be shown that under such a severe confinement, gas molecules can be a dominant factor of liquid motion, and the gas-liquid interaction is significantly time-dependent.

\section{Results}

The molecular-sized nanopores in this study was provided by the hydrophobic nanoporous zeolite $\beta$ (BEA framework type). The average pore size was $0.5-0.7 \mathrm{~nm}^{20}$, in which a single-file molecule chain could be 
formed ${ }^{9,19}$. The specific surface area and pore volume were measured to be $422.60 \mathrm{~m}^{2} / \mathrm{g}$ and $136.10 \mathrm{~mm}^{3} / \mathrm{g}$ by Quantachrome AutosorbiQ-MP gas sorption analyzer. $36 \mathrm{~g}$ zeolite $\beta$ was immersed in $60 \mathrm{~g}$ deionized water, forming a gel-like zeolite $\beta /$ water system, then sealed in a stainless steel chamber. A series of pressure-induced infiltration tests were conducted by using CSS-2220 MTS (material testing system). A piston was compressed into the chamber at various loading rates $(r=0.5-50 \mathrm{~mm} / \mathrm{min})$. Once the pressure reached $120 \mathrm{MPa}$, the piston moved back at the same velocity. Each sample was compressed with five successive loading-unloading cycles.

Figure 1(a) shows the $1^{\text {st }}$ cycle of $P-\Delta V$ curves (pressure-specific volume change curves) at various loading rates. Initially, water molecules cannot enter the hydrophobic nanopores and $P$ changes linearly with $\Delta V$. As it goes to around $25 \mathrm{MPa}$, water molecules enter nanopores and an infiltration plateau is formed. Once all nanopores are filled, the system compressibility reduces abruptly, characterized by the bulk modulus of water. Upon unloading, the invaded water molecules have different degrees of outflow at various loading rates. Water molecules tend to remain inside nanopores at lower $r$ while prefer outflow at higher $r$. This finding serves as an important supplement of Eroshenko's work in $2001^{4}$, in which zeolite $\beta$ /water system is presented as a completely non-outflow system.

Figure 1(b) and (c) show the 5 cycles of $P-\Delta V$ curves at $0.5 \mathrm{~mm} /$ $\mathrm{min}$ and $50 \mathrm{~mm} / \mathrm{min}$ respectively. For $r=0.5 \mathrm{~mm} / \mathrm{min}$, all accessible nanopore space is permanently occupied by the invaded water molecules during the $1^{\text {st }}$ cycle, thus no infiltration is allowed in subsequent cycles. As a consequence, the $P-\Delta V$ curves for the $2^{\text {nd }}-5^{\text {th }}$ cycles are linear in Figure 1(b). While for $r=50 \mathrm{~mm} / \mathrm{min}$, the intruded water molecules will flow out spontaneously once pressure is removed, thus the 5 cycles in Figure 1(c) have similar $P$ - $\Delta V$ curves with plateaus. These findings suggest that zeolite $\beta /$ water system behaves like a reusable liquid spring at higher loading rate, but a disposable energy absorber at lower rate (with an energy density of $3.74 \mathrm{~J} / \mathrm{g}$ for the present system). The residual specific volume $\left(\Delta V_{\mathrm{r}}\right)$ of each cycle is recorded in Figure 1(d), as a quantitative measurement of residual water molecules inside nanopores as the pressure is reduced to zero. It can be observed that $\Delta V_{\mathrm{r}}$ of the $1^{\text {st }}$ cycle decreases with loading rate, being $103 \mathrm{~mm}^{3} / \mathrm{g}$ at $0.5 \mathrm{~mm} / \mathrm{min}$ (close to the specific pore volume $136 \mathrm{~mm}^{3} / \mathrm{g}$ ) and $12 \mathrm{~mm}^{3} / \mathrm{g}$ at $50 \mathrm{~mm} / \mathrm{min}$.


Figure 1 Experimental results of the zeolite $\beta$ /water system at various loading rates $(\boldsymbol{r}=\mathbf{0 . 5}-50 \mathrm{~mm} / \mathrm{min})$. (a) The $1^{\text {st }}$ cycle of $P-\Delta V$ curves at various loading rates. (b) 5 cycles of $P-\Delta V$ curves at $0.5 \mathrm{~mm} / \mathrm{min}$. (c) 5 cycles of $P-\Delta V$ curves at $50 \mathrm{~mm} / \mathrm{min}$. (d) The residual specific volume $\left(\Delta V_{\mathrm{r}}\right)$ of 5 cycles at various loading rates.
Besides, $\Delta V_{\mathrm{r}}$ decreases sharply with cycles at lower rate, while keeps equivalent for each cycle at higher rate.

The above rate-dependent outflow behavior is attributed to the gas-liquid interaction in nanopores. Although currently there's no way to directly observe gas molecules inside such molecular-sized nanopores, it's possible to visually capture the gas phase that has been diffused into the bulk liquid outside the nanopores. Figure 2 shows the snapshots of the zeolite $\beta /$ water system after the $1^{\text {st }}$ cycle at (a) $0.5 \mathrm{~mm} / \mathrm{min}$ and (b) $50 \mathrm{~mm} / \mathrm{min}$. Large amount of air bubbles are observed in (a) while not in (b), indicating that gas molecules can diffuse out and grow into air bubbles at a lower loading rate, while remain confined inside nanopores at a higher rate.

\section{Discussion}

As aforementioned, gas and water molecules will form a single-file chain inside molecular-sized nanopores, with gas molecules deep inside the channels and water molecules around the open end. This anisotropic molecular distribution is energetically unfavorable and unstable, but the radical confinement provides a high resistance for molecular site exchanges. Gas molecules find it hard to physically bypass water molecules to diffuse out; similarly, water molecules cannot freely go deep into nanopores with the block of gas molecules. But through molecular thermal motion, gas and water molecules at the "interface" will keep colliding with each other with high velocity and frequency in a random way. Thus a gradual gas-liquid molecular site exchange can occur, but in a much slower way than the diffusion process in bulk phase.

As a consequence, for the test at $50 \mathrm{~mm} / \mathrm{min}$, since the duration time (around $20 \mathrm{~s}$ ) is insufficient for molecular site exchanges, gas and water molecules stay separately inside nanopores with a clear "interface". Gas molecules are blocked by water molecules and confined in nanopores, thus no air bubble can be observed in liquid phase. The water molecules inside nanopores are subjected to a driving force of outflow from the pressurized gas nanophase, which explains the near-zero $\Delta V_{\mathrm{r}}$ and the reversibility of the system at $50 \mathrm{~mm} / \mathrm{min}$. While for the test at $0.5 \mathrm{~mm} / \mathrm{min}$, the total duration time (around $25 \mathrm{~min}$ ) is nearly 100 times larger, absolutely enough for gas and water molecules to have a thorough nanoscale diffusion. A homogeneous distribution of gas and water molecules can be formed inside nanopores, and gas molecules can gradually diffuse into the external bulk liquid and finally bubble out as the pressure is removed. The water molecules inside nanopores are in a thermodynamic equilibrium without the driving force for outflow. Consequently, they are permanently locked inside nanopores, contributing to a high $\Delta V_{\mathrm{r}}$.

The above nanoscale time-dependent gas-liquid interaction is further validated by a specially designed high-pressure-resting test. In this test, piston moves down at $50 \mathrm{~mm} / \mathrm{min}$; then it's held at the peak pressure for $23 \mathrm{~min}$; and finally it moves upward at $50 \mathrm{~mm} / \mathrm{min}$ for unloading. Thereby, the total duration time for this test is purposely lengthened to that of normal test at $0.5 \mathrm{~mm} / \mathrm{min}$. At the end of this
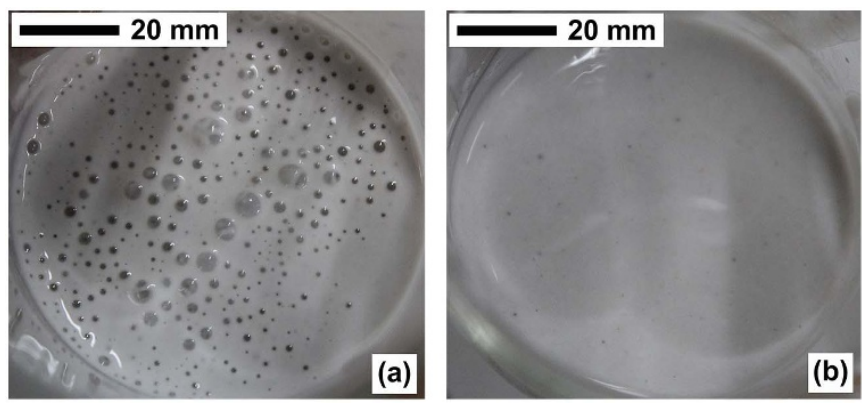

Figure $2 \mid$ Snapshots of zeolite $\beta /$ water system after the $1^{\text {st }}$ cycle at (a) $0.5 \mathrm{~mm} / \mathrm{min}$ and (b) $50 \mathrm{~mm} / \mathrm{min}$. 


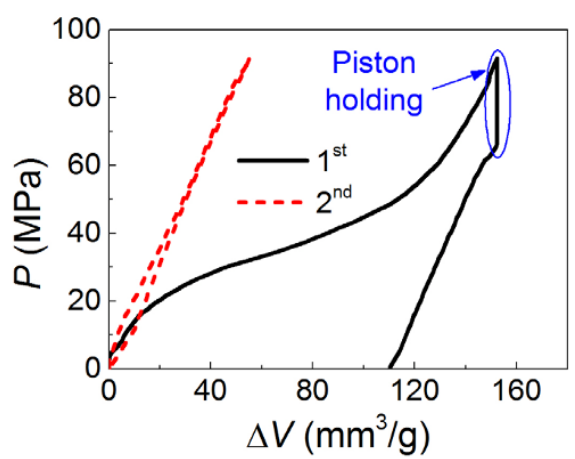

Figure $3 \mid P-\Delta V$ curves of zeolite $\beta /$ water system at $50 \mathrm{~mm} / \mathrm{min}$ with high pressure resting for $23 \mathrm{~min}$.

high-pressure-resting test, large amount of air bubbles are observed and $P-\Delta V$ curve features a high $\Delta V_{\mathrm{r}}$, shown in Figure 3. Not surprisingly, due to the nonouflow of the $1^{\text {st }}$ cycle, there's no infiltration plateau in the $2^{\text {nd }}$ cycle. These results further support the conclusion that the molecular-sized confined gas-liquid interaction is timedependent. Only if enough time is provided for the molecular site exchanges in nanopores, gas molecules can diffuse out and water molecules can remain inside as pressure is removed. The pressure drop during the piston-holding process is in consequence of the nanoscale diffusion. With the homogenization of gas-liquid molecular distribution, the average intermolecular force will decline since the total volume remains unchanged. Besides, since the loading and unloading curve in Figure 3 is identical to that of normal test at $0.5 \mathrm{~mm} / \mathrm{min}$, it can be concluded that the nanoscale liquid-solid interaction is not rate sensitive, and the variation in outflow behavior at different loading rates is exclusively due to the time-dependent gas-liquid interaction.

The significant effect of gas-liquid interaction is peculiar to molecular-sized nanopores. According to our previous work on mesoporous silica with a pore size of $7.8 \mathrm{~nm}$, the gas effect on liquid motion is much weaker in larger nanopores ${ }^{17}$. The mesoporous silica shows a non-outflow behavior independent of loading rate, indicating that both gas and liquid molecules can be "locked" in nanopores, as the external pressure is removed. The liquid "outflow" may be promoted by a thermal treatment. While for molecular-sized pores in the current study, gas and liquid molecules cannot coexist inside nanopores as pressure is removed, primarily due to the high effective density of gas nanophase. The liquid "outflow" can be spontaneous. At a higher loading rate, the molecular site exchanges are limited. At a lower rate, water molecules tend to stay inside the nanopores while gas molecules tend to move out. Besides, the nanopore structures, applied pressure, and properties of gas and liquid may also have impact on the molecular-sized confined gas-liquid diffusion, which will be part of our future work.

In summary, the experimental results on zeolite $\beta /$ water system demonstrate the dominant role of gas nanophase in liquid motion in molecular-sized nanoconfinement. Due to the relatively slow molecular site exchanges in molecular-sized nanopores, gas molecules can provide a driving force for the liquid "outflow" as the external pressure is removed. But if sufficient time is provided, the nanoscale gas-liquid diffusion can be completed, and thus, gas molecules would diffuse out and water molecules would remain inside the nanopores. These findings suggest that time dependent gas nanophase effects must be taken into consideration when gas-liquid-solid interactions in molecular-sized confinements are investigated.

\section{Methods}

Material characterization. Zeolite $\beta\left(\left|\mathrm{Na}^{+}{ }_{7}\right|\left[\mathrm{Al}_{7} \mathrm{Si}_{57} \mathrm{O}_{128}\right]\right)$ contains three-

dimensional 12-ring channels with pore diameters of $6.6 \AA \times 6.7 \AA$ and $5.6 \AA \times 5.6$ $\AA^{20}$. It's constructed by $\mathrm{TO}_{4}$ tetrahedra $(\mathrm{T}=\mathrm{Al}, \mathrm{Si})$, with apical oxygen atom shared



Figure $4 \mid$ Schematic of experimental setup.

between adjacent tetrahedra ${ }^{21}$. Zeolite $\beta$ has three mutually intersecting channels, with two straight channels perpendicular to [001] and a tortuous channel which snakes along the $\mathrm{c}$ crystallographic axis ${ }^{22}$. Zeolite $\beta$ is known as a high mechanical strength materials due to their dense crystalline backbone $e^{23}$, thus the deformation of its framework is negligible in this study. In order to eliminate water and possible impurities, zeolite $\beta$ was firstly preheated at $600^{\circ} \mathrm{C}$ for 3 hours and cooled down to room temperature in air. By using Quantachrome Autosorb-iQ-MP gas sorption analyzer, its specific surface area and pore volume were determined to be $422.60 \mathrm{~m}^{2} / \mathrm{g}$ and $136.10 \mathrm{~mm}^{3} / \mathrm{g}$. By using MasterSizer 2000 particle size analyzer, the average particle size was measured to be $9.11 \mu \mathrm{m}$.

Sample preparation. $36 \mathrm{~g}$ zeolite $\beta$ was immersed in $60 \mathrm{~g}$ deionized water, and then sealed in a 316 stainless steel chamber shown in Figure 4 . Zeolite $\beta$ could be homogeneously distributed in water, forming a stable gel-like liquid. The initial volume and height of zeolite $\beta$ /water system were $100 \mathrm{ml}$ and $72 \mathrm{~mm}$ respectively. The chamber was sealed by precisely-fit sealing rings, whose pressure holding ability has been verified ${ }^{24}$. The pressure could be applied by the piston with a diameter of $40 \mathrm{~mm}$.

Experimental procedures. A series of pressure-induced infiltration tests were conducted on zeolite $\beta /$ water system by using CSS-2220 MTS. The piston was compressed into the chamber at various loading rates $(r=0.5-50 \mathrm{~mm} / \mathrm{min})$. Once the pressure reached $120 \mathrm{MPa}(150 \mathrm{kN})$, the piston moved back at the same velocity. The reaction force $F$ and displacement of piston $d$ could be continuously measured. The friction between the sealing rings and the piston proved to be trivial. Each sample was compressed with five successive loading-unloading cycles for a better observation on its outflow performance. The system compliance was deducted via the tests on a reference system composed of neat deionized water. In order to eliminate air bubbles and gain robust testing data, the bottom of the chamber was finely filleted and each test followed a pre-compression of $10 \mathrm{MPa}$. The pressure $P$ is defined as the reaction force $F$ on piston divided by its cross-sectional area $A$, i.e. $P=F / A$. The specific volume change $\Delta V$ is defined as the volume occupied by piston $(A \cdot d)$ per unit mass of zeolite $m$, i.e. $\Delta V=(A \cdot d) / m$, which consists of contributions from linear compression and pressure induced infiltration. For self-comparison purpose, the infiltration pressure was taken as the pressure at the middle point of the transition zone, defined at the point where the local slope equaled to $150 \%$ of the slope in the infiltration plateau. In current study, the infiltration starts at a pressure of $25 \mathrm{MPa}$ The infiltration volume is defined as the pore volume that are filled during the infiltration test, which can be measured by the width of infiltration plateau. The residual specific volume $\left(\Delta V_{\mathrm{r}}\right)$ is defined as the pore volume occupied by water molecules when the pressure is reduced to zero, which can be measured by the horizontal intercept of $P-\Delta V$ curve.

1. Bianco, A., Kostarelos, K. \& Prato, M. Applications of carbon nanotubes in drug delivery. Curr. Opin. Chem. Biol. 9, 674-679 (2005).

2. Kohli, P., Wirtz, M. \& Martin, C. R. Nanotube membrane based biosensors. Electroanalysis 16, 9-18 (2004).

3. Cao, H., Tegenfeldt, J. O., Austin, R. H. \& Chou, S. Y. Gradient nanostructures for interfacing microfluidics and nanofluidics. Appl. Phys. Lett. 81, 3058-3060, doi:doi:http://dx.doi.org/10.1063/1.1515115 (2002).

4. Eroshenko, V., Regis, R. C., Soulard, M. \& Patarin, J. Energetics: A new field of applications for hydrophobic zeolites. J. Am. Chem. Soc. 123, 8129-8130, doi:10.1021/ja011011a (2001).

5. Sun, Y. et al. A candidate of mechanical energy mitigation system: Dynamic and quasi-static behaviors and mechanisms of zeolite $\beta /$ water system. Mater. Des. DOI:10.1016/j.matdes.2014.04.028 (2014) [In press].

6. Qiao, Y., Liu, L. \& Chen, X. Pressurized Liquid in Nanopores: A Modified LaplaceYoung Equation. Nano Lett. 9, 984-988, doi:10.1021/nl8030136 (2009). 
7. Majumder, M., Chopra, N., Andrews, R. \& Hinds, B. J. Nanoscale hydrodynamics: enhanced flow in carbon nanotubes. Nature 438, 44-44 (2005).

8. Kalra, A., Garde, S. \& Hummer, G. Osmotic water transport through carbon nanotube membranes. Proc. Nat. Acad. Sci. U.S.A. 100, 10175-10180 (2003).

9. Hummer, G., Rasaiah, J. C. \& Noworyta, J. P. Water conduction through the hydrophobic channel of a carbon nanotube. Nature 414, 188-190 (2001).

10. Holt, J. K. et al. Fast mass transport through sub-2-nanometer carbon nanotubes. Science 312, 1034-1037 (2006).

11. Sholl, D. S. \& Johnson, J. K. Making high-flux membranes with carbon nanotubes. Science 312, 1003-1004 (2006).

12. Whitby, M. \& Quirke, N. Fluid flow in carbon nanotubes and nanopipes. Nat. Nanotechnol. 2, 87-94 (2007).

13. Chen, X. et al. Nanoscale fluid transport: Size and rate effects. Nano Lett. 8, 2988-2992, doi:10.1021/nl802046b (2008).

14. Han, A. J. \& Qiao, Y. Pressure-induced infiltration of aqueous solutions of multiple promoters in a nanoporous silica. J. Am. Chem. Soc. 128, 10348-10349, doi:10.1021/ja062037a (2006).

15. Naguib, N. et al. Observation of water confined in nanometer channels of closed carbon nanotubes. Nano Lett. 4, 2237-2243 (2004).

16. Rossi, M. P. et al. Environmental scanning electron microscopy study of water in carbon nanopipes. Nano Lett. 4, 989-993 (2004).

17. Han, A., Kong, X. \& Qiao, Y. Pressure induced liquid infiltration in nanopores J. Appl. Phys. 100, 014308 (2006)

18. Qiao, Y., Cao, G. \& Chen, X. Effects of gas molecules on nanofluidic behaviors. J. Am. Chem. Soc. 129, 2355-2359 (2007).

19. Thomas, J. A. \& McGaughey, A. J. Water flow in carbon nanotubes: transition to subcontinuum transport. Phys. Rev. Lett. 102, 184502 (2009).

20. International Zeolite Association. Database of zeolite structure, $<$ http://izasc.ethz. $\mathrm{ch} / \mathrm{fmi} / \mathrm{xsl} / \mathrm{IZA}-\mathrm{SC} / \mathrm{ftc}$ tm.xsl? $-\mathrm{db}=$ Atlas_main $\&$-lay $=\mathrm{tm} \& S T C=*$ BEA\&-find $>$ (2007).

21. Corma, A., Navarro, M. T., Rey, F., Rius, J. \& Valencia, S. Pure polymorph C of zeolite beta synthesized by using framework isomorphous substitution as a structure-directing mechanism. Angew. Chem. 113, 2337-2340 (2001).

22. Higgins, J. et al. The framework topology of zeolite beta. Zeolites 8, 446-452 (1988).
23. Mitra, A. et al. Synthesis and evaluation of pure-silica-zeolite BEA as low dielectric constant material for microprocessors. Ind. Eng. Chem. Res. 43, 2946-2949 (2004).

24. Sun, Y. et al. Experimental Study on Energy Dissipation Characteristics of ZSM-5 Zeolite/Water System. Adv. Eng. Mater. 15, 740-746, doi:10.1002 (2013).

\section{Acknowledgments}

The work is supported by the Tsinghua University under Grant No. 20121080050 and 20132001016, and the National Natural Science Foundation of China under Grant No. 11372164 .

\section{Author contributions}

Y.S. and Y.Q. wrote the main manuscript text; Y.S., P.L. and Y.L. performed the experiments. Y.S., Y.Q. and Y.L. discussed and commented on the experiments and results. All authors reviewed the paper.

\section{Additional information}

Competing financial interests: The authors declare no competing financial interests. How to cite this article: Sun, Y., Li, P., Qiao, Y. \& Li, Y. Time-dependent Gas-liquid Interaction in Molecular-sized Nanopores. Sci. Rep. 4, 6547; DOI:10.1038/srep06547 (2014).

(c) (i) $(-)$ This work is licensed under a Creative Commons Attribution-NonCommercialNoDerivs 4.0 International License. The images or other third party material in this article are included in the article's Creative Commons license, unless indicated otherwise in the credit line; if the material is not included under the Creative Commons license, users will need to obtain permission from the license holder in order to reproduce the material. To view a copy of this license, visit http:// creativecommons.org/licenses/by-nc-nd/4.0/ 